\title{
Análisis del índice de impacto térmico generado en un ambiente subterráneo
}

\section{Analysis of the index of thermal impact generated in an underground environment}

\author{
Yesid Castro-Duque ${ }^{1}$, Jesús Ramón Delgado-Rodríguez ${ }^{2}$, Jhon Jairo Cáceres-Santos ${ }^{3}$
}

Forma de citar: Y. Castro, J. R. Delgado, J. J. Cáceres, "Análisis del índice de impacto térmico generado en un ambiente subterráneo", Respuestas, vol. 19, no. 2, pp. 32-40, 2014.

Recibido:

Noviembre 20 de 2013

Aceptado:

Abril 10 de 2014

\section{Resumen}

Objetivo: En este artículo se realiza el cálculo y análisis del índice por riesgo térmico, y el concepto del Índice Sintetizado de la Temperatura (SRT), que equivale a un promedio del índice de estrés térmico (WBGT), calculado en diferentes frentes de trabajo de una mina subterránea de carbón. Métodos: Los datos de campo incluyeron medidas de variables climatológicas como la Temperatura Seca (TS) y la Humedad Relativa (HR), y el tiempo de exposición de los trabajadores a dichas condiciones; estas medidas fueron tomadas en la Mina de carbón San José. Resultados: se evidenció que aunque el promedio SRT de la mina clasifica el ambiente como cálido moderado aceptable, existen frentes donde el índice de calor que experimentan los trabajadores es alto y riesgoso. Conclusiones: Se logra concluir que la humedad relativa es la variable que más influye en el calentamiento de los ambientes subterráneos de las minas de la región; de igual forma, se concluye que no es suficiente con determinar el WBGT, sino que se hace necesario establecer la sensación térmica que padecen los trabajadores, a partir del cálculo del índice de calor.

Palabras Clave: Calidad ambiental subterránea, confort, estrés térmico, sensación térmica, impacto térmico.

\section{Abstract}

Objective: in this article the calculation and analysis is performed, of thermal risk index and the concept of the Synthesis-Reduced Temperature (SRT), by calculating heat stress (WBGT) in the atmosphere of an underground coalmine. Methods: Field data included measures of climate variables such as Dry Temperature (DT) and the Relative Humidity $(\mathrm{RH})$, and the time of exposure of workers to such conditions; these measures were taken in the Coalmine San Jose. Result: Became apparent that although the average (SRT) of the mine environment be classified as moderately warm acceptable, there fronts 
where the heat index experienced workers is high and risky. Conclusions: It is concluded that the relative humidity is the most influential variable in warm environments of underground mines in the region; similarly, it is concluded that it is not enough determine the WBGT, it is necessary to establish the thermal sensation experienced by workers, from the calculation of the heat index.

Keywords: Environmental quality underground, comfort, thermal stress, thermal sensation, Thermal Impact.

\section{Introducción}

En las minas subterráneas aumenta la temperatura y la humedad relativa, con la profundidad; estas dos variables inciden en el índice de estrés térmico que experimentan los trabajadores [1]. En este estudio se determinó el Índice de Calor que experimentan los trabajadores, calculado en diferentes frentes de trabajo, y a su vez se halló el índice promedio o sintetizado de calor, conocido como SRT. Para lo anterior, se realizaron medidas de la temperatura seca, la humedad relativa, y los tiempos de permanencia de los trabajadores en varios frentes de trabajo mina de carbón San José. Para la captación de los datos se utilizaron termo-higrómetros y psicrómetros que se ubicaron en los diferentes puntos durante cinco minutos hasta que se estabilizara la medida. Con los datos recolectados, se aplicaron las fórmulas y de acuerdo al resultado se determinó el grado de confort al que se encuentran los trabajadores en el sector aforado.

Se logra concluir que el parámetro de mayor incidencia es la humedad relativa, ya que el índice de calor aumenta con el incremento de esta variable, así la temperatura seca se mantenga estable. El SRT o índice promedio general del área evaluada, determinó el ambiente como aceptable; sin embargo, se encontraron algunos frentes con riesgo alto, por lo que se recomienda complementar los estudios de evaluación general, con la medida del índice de calor. Se concluye que además del índice de estrés térmico, la evaluación del confort de los trabajadores, debe involucrar medidas como la temperatura de la piel, y el balance de calor del cuerpo con el medio ambiente.

Fuentes que causan el aumento de la temperatura en el ambiente subterráneo. A medida que se profundizan las labores mineras, aumenta la transferencia de calor al ambiente subterráneo. Los ambientes subterráneos calurosos afectan, la salud física y mental de los trabajadores, la seguridad, la higiene y la productividad [2].

El aire que entra a un ambiente subterráneo sufre un aumento gradual de su temperatura; dicho aumento, es causado principalmente por: la transferencia de calor de la roca virgen debido al conocido gradiente geotérmico, la auto-compresión, el calentamiento por equipos diésel, la detonación de cargas explosivas, la presencia de aguas termales y el metabolismo humano. [3], [4]. Dos aspectos principales se deben tener en cuenta debido a las altas temperaturas: uno es la afectación de la salud corporal de los mineros, y el otro es el impacto en la seguridad y la productividad de la mina. [3], [5].

-Afectación de la salud corporal. Cuando en un ambiente subterráneo, la humedad y las temperaturas son altas, los trabajadores expuestos a estas condiciones climatológicas, padecen sensación de no confort. Estas 
condiciones pueden ocasionar en los mineros, altos niveles de estrés, aumentado la posibilidad de incremento de accidentes y el decrecimiento de la productividad. A medida que aumenta la profundidad y aumenta el grado de mecanización, las altas temperaturas y los daños producidos por el calentamiento, se convierten en los mayores problemas asociados a la seguridad, limitando la actividad en las minas de carbón.[6].

-Impacto en la seguridad y la producción de la mina. Cuando hay incremento de la temperatura, se puede producir lo siguiente: bajos rendimientos en las labores, generación de incendios, corrosión de equipos y materiales, reducción de la vida útil de los equipos, e incremento de los índices de accidentalidad.

-Efectos fisiológicos sufridos por el hombre debido al aumento de la temperatura. El metabolismo humano está acompañado por la generación de calor, la temperatura del organismo se mantiene próxima a $36.9^{\circ} \mathrm{C}$; el cuerpo del hombre al estar expuesto a la temperatura ambiente, podrá sentir sensación de frio o calor, debido a los efectos fisiológicos causados por bajas o altas temperaturas [7]. El rendimiento del trabajador al laborar en ambientes calurosos disminuye, lo anterior se puede observar en la Figura 1.

La exposición prolongada a condiciones térmicas desfavorables, conduce inevitablemente al aumento de la temperatura del organismo humano y como consecuencia produce efectos fisiológicos que reducen el rendimiento, hasta provocar la muerte, cuando las temperaturas son superiores a $42 \stackrel{\circ}{-} \mathrm{C}[8]$.

El calor del cuerpo, proviene de la oxidación biológica, producto de la absorción del azúcar, la grasa, la proteína y el oxígeno; además, la temperatura corporal aumenta con la intensidad del trabajo. El calor específico del cuerpo es de $3.48 \mathrm{~kJ} /(\mathrm{kg} \cdot$ o $\mathrm{C})$. Si el peso de un hombre es de $60 \mathrm{~kg}$, la cantidad de calor del cuerpo es de $210 \mathrm{~kJ}$; la temperatura corporal aumentará $1 \stackrel{\mathrm{O}}{\mathrm{C}}$, si se incrementa la actividad física en un ambiente donde la temperatura seca fluctúa entre 29 y $32^{\circ} \mathrm{C}$. [3] . En la tabla $\mathrm{I}$, se presenta el incremento de la temperatura corporal, por aumento de la temperatura del aire en un ambiente subterráneo.

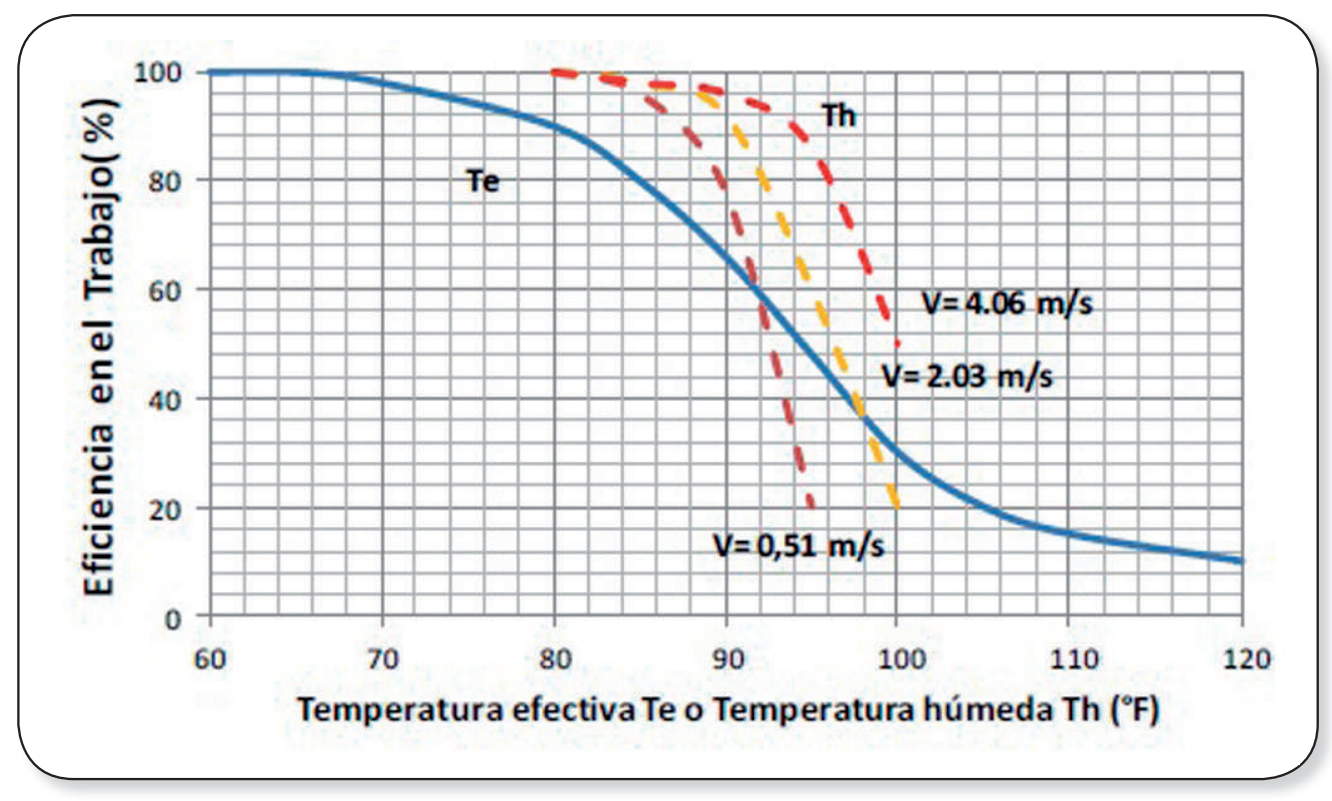

Figura 1. - Efecto de la temperatura sobre el rendimiento del hombre 


\begin{tabular}{|cc|}
\hline Temperatura Húmeda del aire ${ }^{\circ} \mathrm{C}$ & Incremento de la temperatura del cuerpo ${ }^{\circ} \mathrm{C}$ \\
\hline$\leq 29$ & $0.11-0.66$ \\
$29.5-31.7$ & $0.33-0.77$ \\
$32.2-34.5$ & $0.66-1.75$ \\
$\geq 34.5$ & $1.44-1.90$ \\
\hline
\end{tabular}

Fuente: [3]

Antes de sobrevenir el llamado golpe de calor, la persona se siente desorientada, delira, sufre agitaciones y convulsiones. Como consecuencia de esta patología la mayoría de los decesos, se producen dentro de las primeras veinticuatro horas, y en menor escala se dan en un periodo de doce días después de sufrir el golpe de calor [9]. El aumento de la temperatura produce desconcentración en los mineros aumentando el riesgo de accidentalidad; como referente, en la Tabla II, se relaciona el porcentaje de accidentes por aumento de la temperatura del aire, en las minas de Oro de Suráfrica [10].

Tabla II. Relación entre el índice de accidentalidad y la temperatura en la mina

\begin{tabular}{|lcccc|}
\hline Temperatura del aire en el lugar de trabajo ${ }^{\circ} \mathrm{C}$ & 27 & 29 & 31 & 32 \\
\hline Frecuencia de accidentes de trabajo por cada Mil personas & 0 & 150 & 300 & 450 \\
\hline
\end{tabular}

Fuente: [10]

Actualmente se utilizan varios ídices para evaluar las condiciones térmicas a las que se encuentra expuesto el cuerpo humano; los parámetros de dichos índices incluyen medidas de: temperaturas seca, húmeda y efectiva, del bulbo húmedo y Temperatura de globo (WBGT) y medida del estrés térmico (HSI). Sin embargo, algunos de estos índices tienen falencias por la falta de bases teóricas . Por ejemplo, la norma China sobre seguridad para minería del carbón, establece que la temperatura de bulbo seco en el ambiente de un frente de trabajo no debe ser superior a $28^{\circ} \mathrm{C}$, temperatura determinada a partir de la experiencia práctica de trabajo; además, ninguno de los parámetros anteriores, puede exhaustivamente reflejar el estado térmico de los mineros. Las medidas, de temperaturas seca, húmeda y efectiva, del WBGT y del HSI, tienden a centrarse en las condiciones ambientales sin considerar el estado térmico real, el ritmo del corazón o la temperatura del cuerpo, sólo muestran las condiciones de exposicición del cuerpo humano, sin tener en cuenta la interacción de la piel humana o la respiración con el medio ambiente [1].

Marco Legal. La normativa, respecto a los límites permisibles de temperatura que debe haber en un ambiente subterráneo, varía en los diferentes países con tradición minera. En la Tabla III se detallan los valores límites permisibles para algunos países.

Tabla III. Valores límites permisibles de temperatura de algunos países mineros.

\begin{tabular}{|lccc|}
\hline \multicolumn{1}{|c}{ País } & Temperatura seca ${ }^{\circ} \mathrm{C}$ & País & Temperatura seca ${ }^{\circ} \mathrm{C}$ \\
\hline U.S.A. & 30 & Francia & $28 \mathrm{Te}$ \\
Australia & 27 & Sur África & 27.5 \\
Bélgica & $30 \mathrm{Te}$ & Brasil & 30 \\
Portugal & 31 & Zambia & 32 \\
\hline
\end{tabular}


En Colombia el decreto 1335 de 1987, Reglamento de Seguridad en las Labores Subterráneas, estipula en el Título X Capítulo III, lo referente a Climatología. En el artículo 179 se define la temperatura efectiva en un frente de trabajo. El artículo 180 trata sobre los tiempos de permanencia de los trabajadores en los frentes de trabajo de acuerdo a la temperatura efectiva [11]. Tabla IV.

Tabla IV. Tiempo de permanencia máxima.

\begin{tabular}{|cc|}
\hline Temperatura efectiva $\mathrm{Te}^{\circ} \mathrm{C}$ & Tiempo de permanencia en horas \\
\hline 28 & sin límite \\
29 & seis \\
30 & cuatro \\
31 & dos \\
32 & cero \\
\hline
\end{tabular}

Fuente: [11]

Evaluación del riesgo por índice de estrés térmico. El WBGT, es un índice experimental ampliamente usado en la evaluación del grado de riesgo por calor en minas; para calcularlo, se miden las temperaturas seca Ts, húmeda Th y del globo Tg. Este método sintetiza 4 factores, temperaturas, humedad relativa, velocidad del aire y radiación de calor [3]. El WBGT expresado en ${ }^{\circ} \mathrm{C}$, se puede calcular mediante la Ecuación 1, utilizada para ambientes internos:

$$
\mathrm{WBGT}=0,7 \mathrm{Th}+0.3 \mathrm{Tg}
$$

Donde:

- Th, es la temperatura húmeda del ambiente. ${ }^{\circ} \mathrm{C}$

- $\mathrm{Tg}$, es la temperatura del globo. ${ }^{\circ} \mathrm{C}$

Existe una segunda alternativa, sumamente útil cuando no se cuenta con el dato de temperatura de globo negro. Ante eso se debe utilizar la Ecuación 2.de Gagge y Nishi (1976) [12].

Donde:

- Ts, es la temperatura de bulbo seco $\left({ }^{\circ} \mathrm{C}\right)$

- Pa, es la presión o tensión de vapor (Torr.)

Como la tensión de vapor se reporta generalmente en hecto-Pascales, entonces es necesario hacer la Conversión tomando en cuenta que 1 Torr $=133.3224$, entonces la

$\mathrm{Pa}=1.333224 \mathrm{hPa}$. Además, resulta preciso advertir que todavía algunos libros de texto y otras fuentes utilizan la fórmula equivocada de $\mathrm{WBGT}=0.567 \mathrm{Tbs}+0.393 \mathrm{~Pa}+3.94$, error generado en la década de los años 80 y el cual exagera el verdadero índice de estrés por calor ambiental [12].

Para la obtención de la Presión o tensión de vapor, se puede utilizar la Ecuación 3.

$$
\mathrm{Pa}=6,11\left\{10,0^{\wedge}[7,5 \mathrm{~T} /(237,7+\mathrm{D})]\right\}
$$

Donde:

Pa, es la Presión de vapor en Hpa : T es la Temperatura Seca en ${ }^{\circ} \mathrm{C}$; D es la Temperatura de rocío en ${ }^{\circ} \mathrm{C}$.

Para determinar la temperatura de rocío se utiliza la Ecuación 4.

$$
D=T+35 \log H R
$$

Debido a que en la minas, hay varios frentes de trabajo, el WBGT no puede tomarse como índice general para evaluar el riesgo térmico; por lo anterior, la evaluación se puede mejorar con el índice sintetizado de temperatura SRT, Ecuación 5 [3].

$$
S R T=\frac{(W B G T 1) t 1+(W B G T 2) t 2+\cdots(W B G T n) t n}{(t 1+t 2+\cdots t n)}
$$

Donde:

- WBGT, es el índice medido en el 
frente de trabajo 1

- $\mathrm{WBGT}_{2}$ es el índice medido en el frente de trabajo 2.

- $\mathrm{WBGT}_{n}$ es el índice medido en el frente de trabajo $n$

- $t_{1}, t_{2}, t_{n}$ son los tiempos de exposición o de laboreo en minutos

En la Tabla V, se detalla la sensación térmica que pueden experimentar los mineros y el grado de severidad determinado a partir del SRT calculado.

Tabla V. Evaluación del grado de riesgo por calentamiento.

\begin{tabular}{|ccc|}
\hline SRT $^{\circ} \mathrm{C}$ & Sensación térmica & Grado de severidad \\
\hline$>35$ & Muy caliente, no aceptable & Muy severo \\
$32-35$ & Caliente, no confort & Severo \\
$28-32$ & Cálido, aceptable & Moderado \\
$25-28$ & Un poco caliente, Confort & Apacible \\
$23-25$ & Confort, Aceptable & Sin daños \\
$<23$ & Un poco frío & Sereno \\
\hline
\end{tabular}

Fuente: [3]

Índice de calor. El índice de calor es la temperatura aparente que siente el cuerpo, debido a la combinación de los efectos de la temperatura y la humedad. Esto se debe a que transpiración del cuerpo o sudor, no se puede evaporar tan bien cuando la humedad aumenta. Esto ocasiona que los efectos refrescantes del sudor disminuyan conforme la humedad aumenta, lo que trae como consecuencia que el cuerpo no se pueda enfriar de manera natural.

\section{Materiales y métodos}

Para este estudio se realizaron los aforos de temperaturas y humedad relativa con la ayuda del sicrómetro eléctrico PSYCHRO DIAL y el termo-higrómetro EXTECH 45170; estas medidas se efectuaron el día 6 de Abril de 2011, en el sector Inclinado 7 de la Mina San José, la cual ubicada en el cerro Tasajero, al Norte del perímetro urbano de la ciudad de Cúcuta, y cuyo titular es el Consorcio Minero de Cúcuta. En la Tabla VI, se plasman las coordenadas y características de la Bocamina principal.

Tabla VI. Coordenadas y características topográficas del inclinado 7 mina San José

\begin{tabular}{|c|c|c|c|c|c|}
\hline \multirow{2}{*}{ Inclinado } & \multicolumn{2}{|c|}{ Coordenadas } & Altura & Longitud & \multirow{2}{*}{ Dirección } \\
\cline { 2 - 5 } & $\mathrm{X}$ & $\mathrm{Y}$ & m.s.n.m & $(\mathrm{m})$ & \\
\hline 7 & 1376994,66 & 848015,01 & 742,08 & 320 & \multirow{2}{*}{$\mathrm{N} 64^{0} \mathrm{~W}$} \\
\hline
\end{tabular}

Fuente: [15]

\section{Resultados y análisis}

En la tabla VII se presentan los resultados de la evaluación del índice de estrés térmico WBGT, obtenidos a partir de la Ecuación 2; de igual manera, se determinó el promedio o índice general SRT mediante la Ecuación 5. El resultado del SRT para la fecha señalada fue de $28,8^{\circ} \mathrm{C}$; basados en la tabla III, el ambiente de la mina se clasifica como: cálido moderado aceptable. 
Vol. 19

No. 2

Julio-Diciembre 2014 ISSN 0122-820X

PP: $32-40$

Tabla VII. Resultados del cálculo del índice de estrés térmico wbgt.

\begin{tabular}{|c|c|c|c|c|c|c|c|c|c|c|c|c|c|c|c|c|}
\hline Localización & $\mathrm{T} 1$ & $\mathrm{~T} 2$ & T3 & T4 & T5 & T6 & $\mathrm{T} 7$ & T8 & T9 & T10 & T11 & T12 & T13 & T14 & T15 & T16 \\
\hline $\begin{array}{l}\text { Hora } \\
10: 18\end{array}$ & $7: 49$ & $8: 02$ & $8: 15$ & $8: 26$ & $8: 36$ & $8: 4$ & & & $9: 02$ & $9: 11$ & $9: 37$ & 10:09 & $7: 56$ & $8: 54$ & $9: 16$ & $9: 24$ \\
\hline $\mathrm{Ts}{ }^{\circ} \mathrm{C}$ & 26.3 & 29 & 29.5 & 30 & 30.5 & 31 & 30.5 & 31 & 31 & 30.5 & 31 & 28 & 31 & 31 & 30.5 & 31 \\
\hline $\mathrm{Th}{ }^{\circ} \mathrm{C}$ & 24 & 27 & 27.5 & 27.5 & 28.5 & 28.5 & 28 & 28.8 & 28 & 28 & 29 & 26 & 28 & 28 & 27.5 & 27 \\
\hline$\% \mathrm{HR}$ & 83 & 89 & 88.5 & 84 & 88 & 83 & 84 & 85.5 & 85 & 84 & 88 & 84.5 & 83 & 81 & 79 & 74.5 \\
\hline $\mathrm{D}^{\circ} \mathrm{C}$ & 23.5 & 27.2 & 27.6 & 27.3 & 28.6 & 28.2 & 27.8 & 28.6 & 28.5 & 27.8 & 29.1 & 25.4 & 28.2 & 27.8 & 26,9 & 26.5 \\
\hline Pa Torr. & 26.1 & 30.4 & 31.3 & 32.4 & 33.2 & 34.4 & 33.4 & 34.3 & 34.3 & 33.4 & 34.2 & 28.9 & 34.4 & 34.5 & 33.6 & 34.8 \\
\hline WBGT $^{\circ} \mathrm{C}$ & 25.8 & 28.6 & 29.1 & 29.7 & 30.2 & 30.9 & 30.3 & 30.8 & 30.8 & 30.3 & 30.8 & 27.6 & 30.9 & 30.9 & 30.4 & 31.0 \\
\hline $\mathrm{t}$ Min & 480 & 10 & 10 & 5 & 10 & 10 & 5 & 10 & 10 & 5 & 480 & 360 & 10 & 10 & 5 & 360 \\
\hline
\end{tabular}

Nomenclatura: T1-T16. Frentes de trabajo, Ts. Temperatura seca, Th. temperatura húmeda, \%HR. Humedad relativa, D. temperatura de Rocío, Pa. Presión de vapor, t tiempo de exposición.

Fuente: Autores

Aunque con el índice general SRT, se clasificó el confort de la mina como aceptable, hay frentes muy calurosos donde se hace necesario complementar la evaluación con la determinación del índice de calor; con este índice se establece la sensación térmica a la que están expuestos los trabajadores; este parámetro se calculó con el software heat index calculator [13]. En la Tabla VIII y en la figura 3 , se muestran los resultados del cálculo de la sensación térmica.

Tabla VIII. Índice de calor

\begin{tabular}{|c|c|c|c|c|c|c|c|c|c|c|c|c|c|c|c|c|}
\hline Localización & $\mathrm{T} 1$ & $\mathrm{~T} 2$ & T3 & T4 & T5 & T6 & $\mathrm{T} 7$ & T8 & T9 & T10 & T11 & T12 & T13 & T14 & T15 & T16 \\
\hline Hora & $7: 49$ & $8: 02$ & $8: 15$ & $8: 26$ & $8: 36$ & $8: 42$ & $8: 50$ & 9:02 & $9: 11$ & $9: 37$ & $10: 09$ & $7: 56$ & $8: 54$ & $9: 16$ & $9: 24$ & $10: 18$ \\
\hline Ts ${ }^{\circ} \mathrm{C}$ & 26.3 & 29 & 29.5 & 30 & 30.5 & 31 & 30.5 & 31 & 31 & 30.5 & 31 & 28 & 31 & 31 & 30.5 & 31 \\
\hline$\% \mathrm{HR}$ & 83 & 89 & 88.5 & 84 & 88 & 83 & 84 & 85.5 & 85 & 84 & 88 & 84.5 & 83 & 81 & 79 & 74.5 \\
\hline Índice de Calor & 28 & 37 & 39 & 39 & 42 & 42 & 41 & 43 & 43 & 41 & 44 & 33 & 42 & 41 & 39 & 39 \\
\hline Severidad & 1 & 2 & 2 & 2 & 3 & 3 & 2 & 3 & 3 & 2 & 3 & 2 & 3 & 2 & 2 & 2 \\
\hline
\end{tabular}

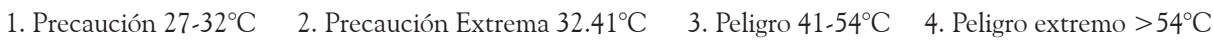
Fuente: Autores

De acuerdo a la tabla anterior, se puede evidenciar que en varios de los frentes, los trabajadores están sometidos a índices de calor riesgosos. Si se hace una comparación entre los frentes de trabajo T11 y T13, en los cuales la temperatura seca $(\mathrm{Ts})$ es igual $\left(31^{\circ} \mathrm{C}\right)$; se nota que en el P11, el índice de calor es $2^{\circ} \mathrm{C}$ más elevado que en el punto de comparación, esto se debe a que en ese frente la humedad relativa fue más alta.

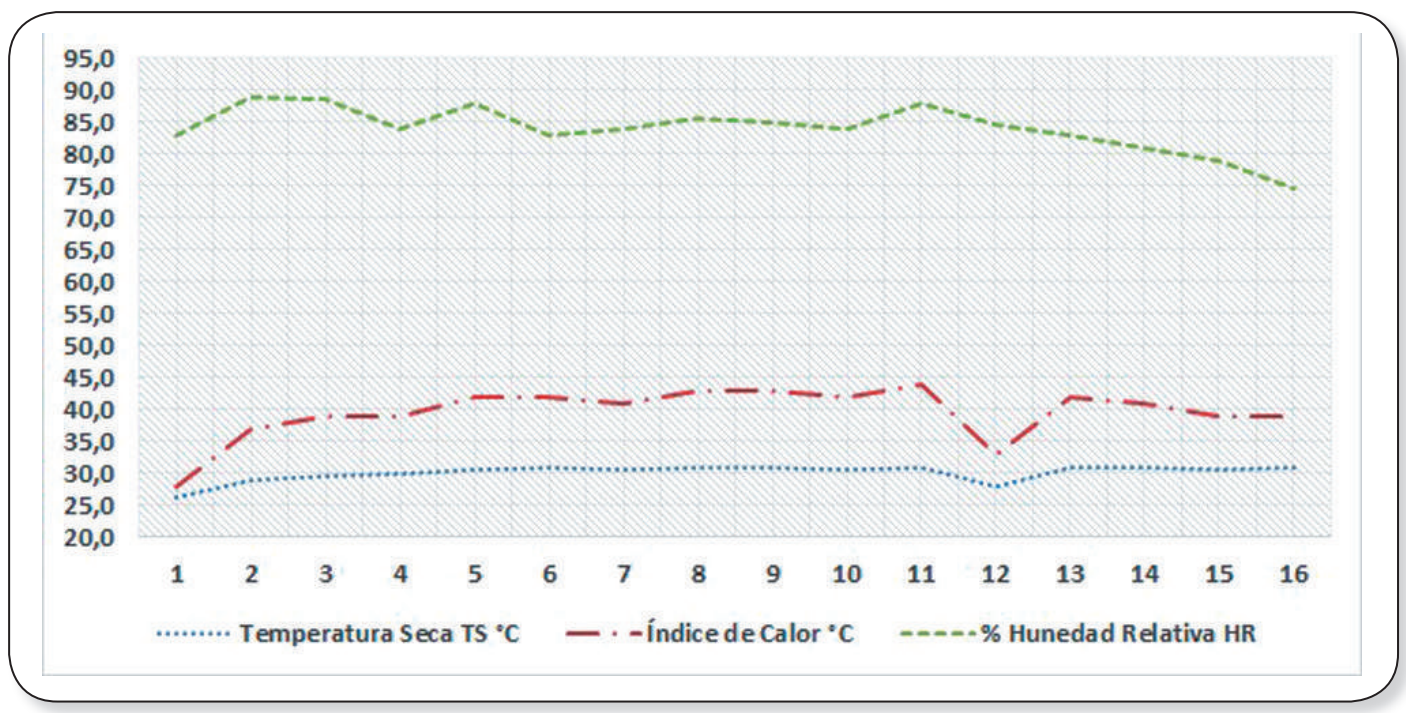

Figura 2. Variación del índice de calor con relación a la Ts y \% Hr

Fuente: Autores 
Resumiendo y basados en los resultados plasmados en la figura 2, se observa que el índice de calor aumenta cuando la humedad relativa es alta, así la temperatura seca se mantenga constante.

Para la obtención de resultados más precisos, los empresarios mineros deben contratar o realizar estudios que involucren el análisis de los diferentes índices, complementados con datos obtenidos a partir de diagnósticos médicos, como: la temperatura corporal y el ritmo de frecuencia cardiaca y ventilatoria de los trabajadores.

Por otro lado, se pueden plantear algunas medidas preventivas y transitorias, como: la rotación de los mineros en los frentes de trabajo, y la disminución de los tiempos de exposición. La más importante medida correctiva para mitigar el impacto térmico, es hacer descender la humedad relativa en las minas, con la implementación de sistemas de ventilación principal mecanizados y la optimización de los sistemas de ventilación auxiliares. De igual forma, los titulares mineros deben equipar sus departamentos de Seguridad e higiene con equipos de medición como Termo-higrómetros, barómetros, y detectores del índice de estrés térmico, para agilizar la captación de datos y la toma de decisiones.

\section{Conclusiones}

Los altos índices de calor, se deben principalmente a la falta de una ventilación principal mecanizada; la mayoría de minas de carbón de la región al igual que la mina evaluada, se ventilan por tiro natural. La ventilación auxiliar para los frentes de trabajo no es óptima ni suficiente por ello el impacto térmico generado es alto.

De acuerdo a los resultados de evaluación del WBGT y del SRT, el área evaluada presenta un ambiente de confort aceptable; sin embargo, al reevaluar el análisis con la determinación del índice de calor, se determina que el riesgo es alto.
En el 38\% de los frentes evaluados, existe un riesgo alto por índice de calor y en un solo frente de trabajo, el tiempo de exposición de los trabajadores a dicho riesgo es alto (480 $\min$ ).

Según [1] cuando la humedad relativa alcanza el 90\%, el comportamiento del metabolismo humano es normal si la temperatura seca no excede los $28^{\circ} \mathrm{C}$; si comparamos estos rangos, con las medidas realizadas en la mina San José, donde se detectaron humedades relativas cercanas al $90 \%$ con temperaturas secas hasta de $31^{\circ} \mathrm{C}$, se concluye que el ambiente se torna riesgoso por el aumento de la temperatura corporal que experimentan los mineros. Por lo anterior, en ambientes subterráneos, si la

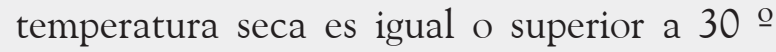
C y la humedad relativa es casi saturada, se hace necesario mejorar y actualizar los índices existentes, así como los criterios y límites que se adoptan para su evaluación. Lo anterior es aplicable a la mayoría de minas subterráneas de la región, en las cuales hay frentes de trabajo donde se presentan temperaturas que oscilan entre 28 y $32{ }^{\circ} \mathrm{C}$, con humedades relativas hasta del $100 \%$.

De acuerdo a la evaluación y los resultados de este estudio, no basta solo calcular el índice de estrés térmico general de una mina; también, se hace necesario determinar el índice de calor al que están sometidos los trabajadores.

Se sugiere, que para otros estudios, los índices se complementen con medidas de la temperatura corporal, talla, peso y frecuencia cardio-respiratoria de los trabajadores.

Los trabajadores de minas subterráneas están sometidos a ambientes no confortables, los cuales les pueden generar estrés por calor ambiental; se recomiendan medidas como: la rotación de puestos de trabajo, y la optimización de los sistemas de ventilación.

Debido a que en el Decreto 1335/87 "Reglamento de Seguridad en labores Subterráneas", no se estipula ninguna limitante en cuanto a la Humedad relativa; se recomienda a los funcionarios de las
Julio-Diciembre 2014 ISSN 0122-820X PP: $32-40$ 
No. 2

Julio-Diciembre 2014 ISSN 0122-820X

PP: $32-40$
Administradoras de riesgos laborales, tener en cuenta normas internacionales para una acertada evaluación del riesgo por impacto térmico en minas subterráneas.

\section{Referencias}

[1] S. Wang, T. Ren, T. Zhang, Y. Liang and $\mathrm{Z}$. $\mathrm{Xu}$, "Hot environment - estimation of thermal comfort in deep underground mines", $12^{\text {th }}$ Coal Operators' Conference, University of Wollongong \& the Australasian Institute of Mining and metallurgy, pp. 241-248, 2012.

[2] Z. Xie, "Distribution law of high temperature mine's thermal environment parameters and study of heat damage's causes", Procedia Engineering,vol. 43, pp. 588-593, 2012.

[3] Z. G. Su, Z. A. Jiang and Z. Q. Sun, "Study on the heat hazard of deep exploitation in high-temperature mines and its evaluation index." Procedia Earth and Planetary Science, vol. 1, no. 1, pp. 414-419, Depth, 2009.

[4] V.F. Navarro Torres, C.D. Da Gama and R.N. Singh, "Mathematical modelling of thermal state in underground mining". Acta geodyn. geomater., vol. 5, no. 4 (152), pp. 341-349, 2008.

[5] W. Bialy, "Environmental working conditions versus occupational diseases in the hard coal mining branch". Scientific Journals Maritime University of Szczecin, vol. 31(103), pp. 37-44, 2012.
[8] J. Yanqiang, and H. Qizong, "Hot Environmental Engineering in Mine", Wuhan Industrial University Publisher, pp. 9-14, 1989.

[9] A. D. Águila, "Procedimiento de Evaluación de Riesgos”, p 22-33, 2005.

[10] C. Anguo. "Formation and Harmfulness of Heat Hazard in Mine and its Control Measure". China Safety Science Journal, vol. 8, pp. 3-5, 2004.

[11] Ministerio de Minas y Energía. Reglamento de Seguridad en labores subterráneas. Decreto 1335/87

[12] L.F. Aragón Vargas, "Nota técnica: el estrés por calor ambiental en el fútbol aumenta en superficies sintéticas" Pensar en movimiento vol. 10 , no. 1, pp. 1-4, 2012.

[13] National Weather service, Weather prediction Center. Software Heat index calculator. 5830 University Research Court College Park, Maryland 20740. 2013.

[14] V. F Navarro Torres, C. Dinis Da Gamma, "Contaminação da atmosfera subterrânea". En Engenharia ambiental subterrânea e aplicações, R. C. Villas Bôas. Rio de Janeiro: CETEM/CYTED, p.81, 92, 2005.

[15] Consorcio Minero de Cúcuta PTO Mina San José, 2011.
[6] X. Song, and Z. Xie, "Research on Mine Cooling Measures for Zhangshuanglou Coal Mine." Procedia Engineering, vol 26(0), pp. 1391-1397, 2011.

[7] V. F. Navarro Torres, "Engenharia ambiental subterrânea e as suas Aplicações a minas Portuguesas e Peruanas." Tesis de Doctorado Instituto Superior Técnico de la Universidad Técnica de Lisboa. 2005. 